\title{
Esprit sociologique et relativisme moral
}

\section{Jacques Coenen-Huther}

\section{OpenEdition \\ Journals}

Édition électronique

URL : http://journals.openedition.org/ress/550

DOI : $10.4000 /$ ress.550

ISSN : 1663-4446

\section{Éditeur}

Librairie Droz

\section{Édition imprimée}

Date de publication : 1 septembre 2003

Pagination : 169-180

ISBN : 2-600-00878-0

ISSN : 0048-8046

Référence électronique

Jacques Coenen-Huther, "Esprit sociologique et relativisme moral », Revue européenne des sciences sociales [En ligne], XLI-126 | 2003, mis en ligne le 30 novembre 2009, consulté le 03 mai 2019. URL : http://journals.openedition.org/ress/550; DOI : 10.4000/ress.550 


\section{Jacques COENEN-HUTHER}

\section{ESPRIT SOCIOLOGIQUE ET RELATIVISME MORAL}

La sociologie a une histoire relativement brève mais elle a une très longue préhistoire. On sait qu'une forme de méditation présociologique est consubstantielle à la pensée occidentale depuis l'antiquité grecque. On a coutume de citer à ce sujet Platon et Aristote. De nos jours, la composante totalitaire de la pensée platonicienne a fait pâlir l'influence de La République. La critique poppérienne y a fortement contribué. En revanche, l'œuvre d'Aristote, véhiculée par la pensée thomiste, reste créditée par certains collègues d'une importance fondatrice. Mais s'il faut chercher une lointaine source d'inspiration dans la Grèce antique, c'est plutôt du côté des Sophistes qu'on peut la trouver: ces Sophistes, dont on a pu dire qu'ils représentent «la pensée dissidente de l'Antiquité » (Dupréel, 1932, pp. 3239) et qui ont été crédités du rejet des idola tribus, les explications mythiques ou traditionnelles de leur époque (Bouthoul, 1949, pp. 10-11) ${ }^{1}$. Mais bien au-delà de ces influences lointaines qui remontent au Ve siècle avant l'ère chrétienne, c'est l'ébranlement d'abord - l'effondrement ensuite - de l'ordre social traditionnel qui crée les conditions de l'avènement de la sociologie comme intention de science. Le projet scientifique de la sociologie s'est affirmé dans un climat général de doutes et d'hésitations quant aux fondements de l'ordre social. Tout ce qui allait de soi - étant justifié jusqu'alors par un ordre transcendant - devint objet de discussions, qu'il s'agisse d'exprimer la nostalgie de la stabilité d'autrefois ou d'assumer la permanence du changement. Cette inquiétude - qui est une inquiétude morale - à l'égard des principes de la cohésion sociale fut alimentée par le sentiment de leur relativité. La conscience du caractère localisé et daté des coutumes, des mœurs, des institutions se manifeste dans l'Ancien Régime sur son déclin. C'est ce qu'on pourrait appeler pour faire bref l'esprit des Lettres persanes dont Yves Fricker traite par ailleurs. Un siècle auparavant, la relativité des choses humaines cherchait encore à se camoufler. Dans ses Pensées, Pascal, associant la relativisation à la subversion, écrit que si une coutume «a été introduite autrefois sans raison [...] il faut la faire regarder comme authentique, éternelle, et en cacher le commencement si l'on ne veut qu'elle ne prenne bientôt fin » $(1640,1943$, pp. 173-175). Chez Montesquieu, plus rien ne subsiste de la méfiance pascalienne. Dans L'Esprit des lois, le thème de la relativité s'exprime sans réticence. Ultérieurement, les sociologues ne se firent pas faute d'attirer l'attention sur la diversité des institutions humaines. Celle-ci leur fournit même le fondement d'une

J'ai déjà évoqué le rôle précurseur des Sophistes dans une publication antérieure (Coenen-Huther, 1998, pp. 121-122). 
méthode: l'approche comparative offrant un substitut à la méthode expérimentale. On en arrive ainsi peu à peu à considérer la sociologie comme la discipline relativisante par excellence. Il est d'ailleurs significatif que Protagoras, un des Sophistes de la première génération, puisse être présenté tout à la fois comme l'ancêtre des penseurs postmodernes et comme un des précurseurs lointains de la pensée sociologique. Sa maxime célèbre «L'homme est la mesure de toute chose, de celles qui sont, qu'elles sont; de celles qui ne sont pas, qu'elles ne sont pas » a pu être considérée comme l'annonce d'une «philosophie de la convention» et l'anticipation de l'esprit sociologique (Dupréel, 1932, p. 130). Tocqueville, en son temps, note que «ce qu'on appelle les institutions nécessaires ne sont souvent que les institutions auxquelles on est accoutumé» et qu'en la matière «le champ du possible » est très vaste $(\dagger 1893,1964$, pp. 96-97). Plus tard, l'utilisation même du terme «social» véhiculera souvent un a priori théorique et méthodologique selon lequel des conceptions tenues pour universelles sont en fait relatives, c'est-à-dire limitées à une société donnée, voire même à un sous-groupe de celle-ci, «car leur détermination fait appel au système de valeurs de cette société» (Lemel, 1991, p. 33). Mais l'attitude relativisante poussée à son terme ne peut qu'exercer un effet de sape sur les institutions les plus solidement établies. C'est ce que redoutait Pascal. C'est également ce qu'avait compris Tocqueville pour qui «le champ du possible» se resserrait manifestement lorsqu'il s'agissait du droit de propriété $(\dagger 1893,1964, \text { p. } 37)^{2}$.

Pour la sociologie moderne, évoluant en symbiose avec l'institution universitaire, un problème majeur est de préserver la possibilité d'une perspective relativisante - perspective qui est aux fondements de la pensée sociologique - tout en échappant à ce relativisme paralysant qui inquiète un certain nombre de bons esprits. Les sociologues actifs en milieu universitaire ne peuvent adopter une attitude d'indifférence aux conséquences logiques de leur posture fréquemment relativisante. Ils ne le peuvent parce que ces conséquences logiques sont aussi des conséquences pratiques. Ils participent à une oeuvre éducatrice. Ils transmettent des connaissances, certes, mais - qu'ils le veuillent ou non - ils offrent également des modèles culturels et ils suggèrent par leur propre comportement des normes de conduite. Cela entraîne des responsabilités. Peut-on dès lors être sociologue et jouer en tant que tel un rôle d'agent socialisateur à l'égard de la jeunesse estudiantine? En d'autres termes, est-il possible de rester sensible à la relativité des institutions, des coutumes, des mœurs, etc., tout en défendant par le discours et par l'exemple une échelle de valeurs ferme face aux jeunes gens et aux jeunes filles qui nous écoutent? N'y a-t-il pas là deux rôles aux exigences contradictoires, l'un prenant nécessairement le pas sur l'autre, d'autant plus que les principes éducatifs eux-mêmes se prêtent à des analyses qui en relativisent la portée dans le temps et dans l'espace?

Je ne chercherai pas à cacher que j'ai vécu cette contradiction sur le mode existentiel au cours de mes dernières années à l'Université de Genève. Plus d'une fois, je me suis senti inhibé dans l'exercice de mes responsabilités d'agent socialisateur par la portée relativisante du discours sociologique dont je me faisais le porteparole. C'est ce qui me pousse à explorer la question ici car je suis porté à croire

Voir à ce sujet mon Tocqueville où j'ai fait usage des mêmes citations (1997, p. 16). 
que je ne suis pas le seul à avoir fait l'expérience de cette contradiction. Il n'y a ici aucune énigme à résoudre mais bien une dissonance à réduire. Je crois d'ailleurs, comme Jean-Michel Berthelot, que la notion de «dissonance» caractérise mieux ce qui stimule la réflexion sociologique que le terme d'énigme, plus approprié à l'épistémologie des sciences de la nature (Berthelot, 1996, p. 28). Si cette dissonance n'était pas réduite, il faudrait alors, en toute honnêteté intellectuelle, se poser le problème d'effets pervers éventuels de la connaissance sociologique et de sa diffusion vulgarisée. Au sein de notre corporation, on se demande souvent si la sociologie peut avoir un impact réel sur le traitement des problèmes de société. C'est une interrogation tout à fait respectable ${ }^{3}$. Mais ne faudrait-il pas se demander également si la sociologie n'a pas, dans certains cas, une influence non souhaitable: une influence que nous-mêmes ne souhaitons pas, en tant qu'individus et en tant que citoyens. Dans un livre récent, L'obsession anti-américaine, Jean-François Revel s'en prend avec la verve polémique qu'on lui connaît «aux sociologues politiquement corrects » qui, selon lui, font preuve d'un angélisme proprement désastreux face à la montée de la délinquance et de la violence (2002, p. 176). Ne faudrait-il pas se demander si les sociologues n'ont pas une propension, induite par leurs procédés de raisonnement, à se soucier exagérément - plus que d'autres peut-être - du caractère «politiquement correct» de leurs déclarations. Le souci excessif du «politiquement correct» ne serait-il pas directement lié à la priorité accordée à des variables structurelles, à des variables d'appartenance, qui exonèrent totalement ou partiellement l'individu de la responsabilité de ses actions?

Quoi qu'il en soit, le dilemme de la relativisation et de la transmission légitime de normes ne peut pas ne pas être un objet de préoccupations pour la sociologie. Une solution apparemment séduisante consiste à restaurer l'idée de nature humaine et à mettre en évidence des constantes plongeant leurs racines dans une animalité préexistant à toute forme de culture ${ }^{4}$. Un noyau d'instincts sociaux élémentaires pourrait alors être considéré comme transcendant les époques et les cultures. Le sens moral pourrait ainsi être préservé de tout relativisme nihiliste. Cette conception d'un sens moral ancré dans la nature plutôt que dans la culture paraît s'imposer d'autant plus facilement que les connaissances en éthologie progressent très rapidement de nos jours (Lestel, 2001, p. 15). Nous avons vécu pendant des siècles sur l'idée d'une frontière bien nette entre le genre humain et le reste du règne animal (Ibid.). On nous rappelle à présent que l'humain, caractérisé par un potentiel d'autonomisation culturelle vis-à-vis de la nature, est le produit de l'évolution naturelle des espèces. Cette idée n'est certes pas neuve. Au début $\mathrm{du} \mathrm{XX}^{\mathrm{e}}$ siècle, Miguel de Unamuno, dans Le sentiment tragique de la vie, n'hésitait pas à affirmer que «... l'homme n'est, à la lettre, qu'une espèce de gorille, d'orang-outang, de chimpanzé ou quelque animal voisin, devenu hydrocéphale ou quelque chose d'approchant.» (1917, p. 34). Mais l'idée revient en force à l'heure actuelle (Lestel, 2001, p. 162). Ceci suggère que des stades intermédiaires

J'ai moi-même contribué à plusieurs reprises au débat sur cette question (Coenen-Huther, 1969, 1979, 1987).

$4 \quad$ Voir à ce sujet l'ouvrage de J. Q. Wilson, The Moral Sense (1993) et le commentaire qu'en donne Raymond Boudon dans Le juste et le vrai (1995). 
peuvent exister entre les conduites purement instinctuelles et les comportements marqués par la réflexivité. Le rire est le propre de l'homme, a-t-on pu affirmer longtemps, à la suite de Bergson, sans risquer d'être contredit. Il semble bien que notre cousin le chimpanzé soit en mesure de nous déposséder de l'exclusivité de la perception du comique: des expériences tendent à le montrer (Lawick-Goodall, 1973; Lestel, 2001, pp. 204-205). La vision de l'humain comme produit de l'évolution des espèces nous suggère également que des traces d'animalité subsistent dans les attitudes et les comportements des êtres humains d'aujourd'hui. Mais tout ceci ne doit pas semer la confusion dans les esprits. L'acte moral est un acte réfléchi, volontaire, impliquant un aspect de sacrifice consenti ou, à tout le moins, de détriment volontairement accepté (Ferry et Vincent, 2000, pp. 49 et ss.).

Penser un acte comme acte moral exige l'existence d'un individu qui en ait l'initiative consciente et qui ne se contente pas d'obéir à un instinct ou à un réflexe. Ceci implique une capacité de prise de distance réflexive par rapport à la nature mais aussi par rapport au cours de l'histoire (Ibid., p. 32). Que cette capacité existe à l'état de balbutiement, à l'état d'ébauche et de promesse, chez d'autres espèces vivantes ne lui enlève nullement sa caractéristique de condition sine qua non ${ }^{5}$. Et l'idée que la part d'animalité qui est en nous puisse être le fondement d'un comportement moral est une aberration de l'esprit. La sensibilité éthique, impliquant nécessairement la responsabilité, fait appel à notre potentiel de liberté pour dominer - et s'il le faut pour vaincre - notre naturalité. Que la morale humaine soit un produit de l'évolution, comme le veulent les tenants d'une éthique évolutionniste, n'enlève rien au fait que l'acte moral suppose liberté, responsabilité et capacité de débat avec soi-même. Ce sont là des ingrédients qui sont absents des comportements dits «altruistes» qu'on peut observer chez certaines espèces animales.

Une autre solution - philosophiquement plus acceptable - au problème du relativisme cognitif et moral est celle que nous propose Raymond Boudon depuis une dizaine d'années (Boudon, 1995; 1999). Je ne crois pas trahir sa pensée en disant qu'elle consiste pour l'essentiel à neutraliser le polythéisme des valeurs. Cette neutralisation s'effectue par la relativisation de la distinction désormais classique entre jugements de fait ou de réalité et jugements de valeur. Le normatif est ainsi ramené en dernière instance à du cognitif. Cette façon de voir implique en outre qu'on ancre les convictions morales dans la transsubjectivité. L'acteur individuel éprouve des convictions morales parce qu'il a des raisons solides d'accepter les raisonnements qui peuvent être présentés à l'appui de ces convictions et qui rencontrent l'approbation générale. J'ai déjà eu l'occasion d'indiquer les problèmes que pose à mon avis le recours à la transsubjectivité (Coenen-Huther, 2001b). Je n'ai pas l'intention d'y revenir dans le cadre de cette communication. Ce que je voudrais souligner ici, c'est qu'il me paraît difficile d'apporter une réponse intellectuellement et moralement satisfaisante au conflit de rôles évoqué plus haut, sans une théorie des valeurs solides. Et sur ce point, je reste fidèle à deux de mes maîtres bruxellois, Eugène Dupréel et Chaïm Perelman, dont les influences ne vont pas tarder à apparaître au fil de mon propos.

Comme le rappelle fort opportunément Luc Ferry dans son ouvrage récent Qu'est-ce qu'une vie réussie? (Paris, Grasset, 2002, pp. 418-425). 
Promouvoir une valeur, c'est la poser en principe comme irréductible à d'autres valeurs. Les valeurs n'acquièrent consistance - c'est-à-dire cohérence, fermeté, solidité - qu'en se dégageant d'autres valeurs auxquelles on peut les opposer (Dupréel, 1939; 1949). Dès lors, une valeur est un objet d'accord qui ne peut prétendre à l'adhésion d'un auditoire universel. Au-delà des échelles de priorité des différents groupes sociaux ou culturels, le beau, le bien, le vrai ont été, il est vrai, érigés en valeurs absolues. De telles valeurs ultimes ont certes un maximum de consistance mais elles ont en même temps un maximum de précarité car elles impliquent un accord des esprits qui n'est jamais entièrement réalisé. Pour que les valeurs ultimes puissent être célébrées universellement, il faut qu'on n'en spécifie pas le contenu: «c'est dans la mesure où elles sont vagues » qu'elles peuvent prétendre à l'universalité; précisées davantage, elles ne reflètent plus que «les aspirations de certains groupes particuliers » (Perelman et Olbrecht-Tyteca, 1970, pp. 101-102). Chacune des valeurs absolues - et certainement la valeur du bien qui fonde le jugement moral - s'actualise dans des comportements qui ne prennent leur signification sociale que par rapport à une norme préexistante, qu'il s'agisse de s'y conformer ou au contraire de la refuser. La conformité aux normes est l'attitude qui semble se prêter le mieux à une analyse de type durkheimien ou néo-durkheimien, mettant l'accent sur la pression sociétale. Mais si le fait de la pression contraignante est bien réel, la valeur explicative de ce fait est pauvre. Elle est pauvre, non pas tellement parce que la pression n'est pas uniformément efficace - cet argument ne m'a jamais paru très convaincant - mais parce qu'on peut tout aussi bien renverser la chaîne de causalité et se demander ce qui explique l'existence de la pression (Dupréel, 1932, p. 322). On se condamne sur ce point à énoncer des propositions triviales ou réductrices si l'on perd de vue l'interpénétration constante de l'individuel et du social telle qu'elle a été mise en évidence par la sociologie interactionniste influencée par les conceptions de George Herbert Mead et, dans un registre tout à fait différent, par Marcel Proust, très habile à nous présenter un soi multiple, d'une extrême sensibilité aux influences du milieu et à l'écoulement du temps ${ }^{6}$. La pression sociétale s'exerce sur l'individu mais cette pression est le fait d'une multitude d'individus en interaction. La liberté de l'individu est une liberté sous contrainte mais cette contrainte - qui n'est pas un déterminisme - est le résultat net des actions ou des attitudes conjuguées d'une multitude d'autres individus, également en liberté sous contrainte. La contrainte extérieure n'efface pas la liberté de l'individu: elle en fixe les paramètres limitatifs ou - si l'on préfère - elle la contextualise ${ }^{7}$. Il n'est de liberté que par rapport à des options qui apparaissent au niveau de conscience de l'individu.

C'est donc bien la prise de distance réflexive par rapport à un environnement plus ou moins contraignant - mais aussi par rapport à la tentation forte de se soumettre inconditionnellement aux contraintes de cet environnement - qui est à l'origine de l'acte moral comme expression de l'adhésion à une valeur. L'individu

Au sujet des affinités de l'œuvre de Proust avec la sociologie, voir l'ouvrage très documenté de Pierre Lassave, Sciences sociales et littérature (Paris, PUF, 2002). On peut s'étonner que l'aspect de l'œuvre proustienne évoqué ici ne soit pas celui que les présentateurs de «Proust sociologue» mettent le plus en évidence car c'est certainement le plus original.

7 Et, ce faisant, elle réduit l'ampleur de la «double contingence» signalée par Talcott Parsons et Niklas Luhmann. 
particulièrement sensible à la dimension morale de ses actes est l'individu innerdirected, c'est-à-dire introdéterminé, de David Riesman (1950). On peut présenter les choses différemment en utilisant la notion d'acteur banalisé, expression lancée il y a une vingtaine d'années par Raymond Boudon (1984, p. 22). L'acteur banalisé à dessein pour les besoins de l'analyse sociologique doit être caractérisé, pour reprendre les termes de Simmel dans la Philosophie de l'argent, par une capacité d'objectivation, c'est-à-dire par une capacité de raisonnement faisant provisoirement abstraction des impulsions subjectives de son moi (1907, 1999, pp. 355356). Négliger ce qui caractérise ainsi au plus haut degré l'être humain, c'est s'astreindre à ne pouvoir raisonner que sur la conformité - fût-elle une conformité de marginaux - et s'interdire de prendre en compte la déviance annonciatrice de conformités nouvelles, ce qui est une lacune grave dans une période de changement social aussi rapide que la nôtre. A notre époque précisément, des voix se font entendre pour déplorer le «déclin des valeurs » et ces voix ne sont pas seulement celles de ces «intellectuels-prêcheurs» auxquels Raymond Boudon fait allusion dans un petit ouvrage récent (2002, p. 108). Tout en éprouvant une réelle sympathie pour les sentiments nostalgiques qui s'expriment de la sorte - tout en les éprouvant moi-même -, je ne peux m'abstenir de faire observer que ce discours actuel sur le «déclin des valeurs», pris au pied de la lettre, est sociologiquement absurde. Non pas, parce qu'il semble en contradiction avec des données de sondages d'opinion récents, mais - plus fondamentalement - parce qu'il n'y a pas de groupe social plus ou moins organisé sans valeurs. Le «déclin des valeurs »si déclin des valeurs il y a-n'est rien d'autre que le déclin de valeurs qui nous sont chères et dont la transmission intergénérationnelle nous paraît sérieusement compromise. L'analyse sociologique ne peut que repérer le déclin éventuel de certaines valeurs et l'émergence d'autres valeurs. Par «déclin», il ne faut d'ailleurs rien entendre d'autre que la perte de force normative d'une valeur, sans qu'on puisse à cet égard préjuger de l'avenir.

Mais le sociologue est partie prenante à cette vie sociale qu'il fait profession d'étudier et dont il est l'observateur participant ${ }^{8}$. En tant que participant, il aura tendance à absolutiser ce que l'observateur, au contraire, sera enclin à relativiser. Il sera ainsi aux prises avec un conflit de valeurs irréductible - le vrai et le bien que d'autres époques auraient éliminé par l'adhésion à une vision religieuse du monde. Parmi nos prédécesseurs immédiats, Raymond Aron, homme d'action autant qu'homme d'étude, est un de ceux qui affrontèrent ce conflit de valeurs avec le maximum de lucidité. La position qu’il définissait comme celle du «spectateur engagé » impliquait un exercice de «corde raide» entre la raison et la passion: ambition de faire œuvre de raison, exigeant la prise de distance critique à l'égard de nos propres prénotions, voire de nos propres préjugés, mais aussi attachement ferme à des valeurs que seule peut garantir une certaine dose de passion (1981; 1983, pp. 723-726). L'attachement à une valeur ne peut faire l'objet d'une démonstration. On peut soumettre à la vérification rationnelle le caractère vrai d'une proposition; on ne peut pas imaginer de procédure semblable qui justifie la

J'ai eu l'occasion de suggérer ailleurs que l'observateur participant, loin d'être un chercheur un peu marginal, est au contraire «la figure paradigmatique du sociologue aux prises avec la réalité sociale qui l'entoure» (Coenen-Huther, 1996, p. 134). 
dévotion à la valeur de vérité elle-même. Le polythéisme des valeurs ne débouchait pas, chez Raymond Aron, «sur un relativisme négateur de toute valeur» (Baverez, 1993, p. 510), comme l'imaginait Fauconnet, mais au contraire sur un appel résolu à l'engagement qui, il est vrai, ne s'était pas encore manifesté au moment de la fameuse soutenance de 1938 qui l'opposa à un positivisme d'inspiration durkheimienne (Aron, 1983, pp. 105-106). Comme toute attitude fondée sur un compromis entre des aspirations contradictoires, l'attitude du «spectateur engagé » n'est certainement pas facile à mettre en pratique. Elle entraîne même des dilemmes moraux qui ne peuvent être vécus au plan individuel que sur le mode tragique. C'est ce qui a pu faire dire que le libéralisme d'Aron «[...] est une philosophie pour âmes bien trempées qui, rejetant le confort moral des certitudes idéologiques, acceptent le caractère tragique de l'histoire, partiel de leur savoir, aléatoire du pari de l'humanité sur la raison.» (Baverez, 1993, p. 510). Cette attitude n'en reste pas moins intellectuellement concevable lorsqu'il s'agit de choix politiques fondamentaux comme ceux auxquels Aron fut lui-même confronté: les démocraties contre la barbarie hitlérienne, les régimes pluralistes face au totalitarisme soviétique. Elle peut se traduire alors dans la pratique par un engagement sans réserve, assorti du maximum d'honnêteté intellectuelle vis-à-vis des positions de l'adversaire. Mais le compromis entre la distance intellectuelle et l'engagement dans l'action offre-t-il une recette acceptable lorsqu'il s'agit de l'attitude à adopter face aux jeunes générations? En d'autres termes, se traduit-il par des rôles aux contours bien définis, qui puissent être assumés sans trop de problèmes par un enseignant-chercheur, lorsque celui-ci a pour mission de former de jeunes universitaires et d'enseigner la sociologie?

Essayons de concrétiser les choses. Nous vivons à une époque où le désarroi en matière éducative est manifeste. Il suffit de lire la presse quotidienne et hebdomadaire pour s'en rendre compte. Qu'on en soit arrivé à envisager en Suisse l'introduction d'une «formation à la parentalité» dans les programmes scolaires indique bien l'ampleur d'une crise qui affecte non seulement le système d'enseignement, depuis l'école primaire jusqu'à l'enseignement supérieur, mais aussi le monde familial. Cette crise se manifeste, notamment, par l'affrontement de prises de position contradictoires à propos de ce qu'on convient d'appeler les «droits de l'enfant» et par la confusion entretenue à ce sujet entre «droits-libertés » et «droits-créances». Je m'inspire ici de l'ouvrage d'Alain Renaut, La libération des enfants (2002) bien que je ne puisse adhérer sans réserve à toutes les prises de position de cet auteur'. Les droits-libertés sont des droits que «la théorie générale des droits de l'homme» identifie comme tels, à savoir liberté d'opinion, liberté d'expression, liberté de pensée, de conscience et de religion, liberté d'association, de réunion pacifique [...] droit au respect de la vie privée » (Renaut, 2002, p. 339). Les droits-créances sont les droits qui se réfèrent logiquement «à la fragilité et à l'inachèvement de l'enfant». Il s'agit du «droit de jouir du meilleur état de santé possible », du «droit de bénéficier de la sécurité sociale », du droit «à un niveau de vie suffisant», du «droit à l'éducation», etc. Ces droits-là sont «accompagnés par

En particulier, contrairement à ce que suggère Renaut, je ne pense pas qu'on doive considérer comme inévitable et irréversible le processus actuel d'élargissement de l'idée de démocratie à des domaines où elle n'a que faire. 
un ensemble de protections allant dans le même sens» (Ibid. p. 338). Par une extension tout à fait discutable de l'idée de démocratie - je serais même tenté de dire: par un détournement de l'idée de démocratie -, la culture du débat se répand, donnant l'impression que les bénéficiaires de droits-créances sont nécessairement des sujets de droits-libertés. Dans la relation d'enseignant à enseigné tout comme dans la relation parents-enfants, tout semble offrir matière à discussion, à négociation, à contestation. Les conceptions éducatives qu'on tenait pour solidement acquises font l'objet de remises en question.

Dans ces conditions, l'incitation au respect de l'institution universitaire, la socialisation au métier d'étudiant, la défense de principes élémentaires de discipline, de civilité, de rigueur intellectuelle, peuvent-elles coexister avec le message relativisant de la sociologie? Autrement dit, que faisons-nous quand nous enseignons la sociologie et que nous mettons l'accent sur le caractère socialement construit des mœurs, des coutumes, des institutions? L'expression «construction sociale de la réalité» n'a-t-elle pas déjà été détournée de sa signification initiale pour devenir synonyme d'arbitraire culturel et justifier le rejet individualiste de toute contrainte ? $^{10}$ Pouvons-nous prétendre contribuer à l'édification d'une morale de notre temps, comme l'aurait voulu Durkheim, ou contribuons-nous au contraire, par aveuglement plutôt que par volonté délibérée, à saper les bases d'un ordre social déjà fragilisé? On peut, bien sûr, applaudir à la subversion de l'institution universitaire comme de toute autre institution. Une telle orientation idéologique cherche volontiers sa légitimation dans la fonction critique de la sociologie. Il existe aussi une subversion qui s'ignore ou qui ne s'affiche pas comme telle. Elle est douce, insidieuse; elle prend le visage accueillant du laxisme pédagogique. Mais qu'en est-il de ceux d'entre nous qui se veulent tout à la fois sociologues fidèles à l'intention de science de nos classiques et éducateurs faisant preuve du sens de leurs responsabilités? Pour s'exprimer en toute familiarité, le sociologue qui cherche à initier de jeunes esprits aux fondements de la perspective sociologique n'est-il pas en train de «scier la branche sur laquelle il est assis » en sa qualité d'enseignant? L'observation de la vie universitaire suggère que le problème est ressenti confusément, même si une certaine pudeur empêche d'en parler. Des stratégies d'adaptation plus ou moins cohérentes sont discernables. Aucune n'est pleinement satisfaisante. L'attitude qui me paraît la plus fréquente est fondée sur la ségrégation des rôles la plus classique: on tient ex-cathedra un discours théorique à portée relativisante tout en faisant échapper implicitement ses propres principes d'enseignant à la relativisation et en espérant que personne ne mettra le doigt sur la contradiction. Une autre attitude qui est bien dans l'air du temps est celle de la démission éducative. Elle est très tentante car elle prémunit d'emblée contre toute contestation ou tout reproche d'inconséquence. On s'évade de la relation de maître à élève qui est logiquement une relation asymétrique fondée sur l'autorité et on se désolidarise intellectuellement et moralement de l'institution qu'on représente. On est là, dit-on, pour enseigner une matière et pas pour socialiser à l'institution universitaire, pour préserver la légitimité de la fonction enseignante ou pour maintenir les conditions d'une transmission efficace de

10 C'est ce que j'ai suggéré dans un article consacré à la sociologie des sciences (Coenen-Huther, 2002, p. 128). 
normes de comportement. Autrement dit, on abdique son rôle d'agent socialisateur et l'on s'en tient à une conception minimaliste de son rôle d'enseignant. Une troisième attitude courante est la fuite en avant dans un prétendu avant-gardisme éducatif - plus de sélection, plus de notes, plus d'échecs - dont nos collègues psychologues commencent sérieusement à s'effrayer en raison de la perte de repères qu'il provoque. On fournit ainsi une pseudo-justification sociologique à la mise en œuvre d'innovations pédagogiques dont il est plus facile de déceler les effets pervers que de distinguer les bienfaits.

Cela étant, peut-on énoncer l'un ou l'autre principe général qui offre au sociologue la perspective d'assumer ses choix de valeur autrement que sur le mode schizophrénique? C'est ce que je voudrais tenter pour terminer, avec toute l'humilité qu'impose le sentiment très vif d'abandonner un navire en perdition - l'institution universitaire - et d'avoir été tout aussi impuissant que d'autres à en éviter la dérive ${ }^{11}$. Tout d'abord - cela doit être répété de façon tout à fait dénuée d'ambiguïté - l'esprit sociologique a une composante relativisante indéniable. Cela signifie qu'une vulgate sociologique contribue, que cela plaise ou non, à la diffusion d'une mentalité relativiste. C'est un fait dont tout sociologue a intérêt à prendre conscience et dont il lui appartient de tirer les conclusions pour lui-même. Cela signifie également que le relativisme ambiant n'épargne pas la sociologie institutionnalisée elle-même, d'autant moins que la reconnaissance du caractère pluriparadigmatique de la sociologie alimente le sentiment de la relativité des points de vue et des perspectives. Ceci dit, la conscience de l'enracinement culturel et social des valeurs ne doit pas pousser le sociologue à vouloir tenter l'impossible démonstration du bien fondé de tel ou tel jugement de valeur. Les valeurs ne se démontrent pas, elles s'affirment. Et la nécessité de les présenter sur le mode péremptoire indique tout à la fois leur consistance et leur précarité. Si les choix de valeur ne se démontrent pas, ils s'argumentent, il est vrai. Mais il faut bien voir que les argumentations proposées ne peuvent en aucune façon prétendre rallier un auditoire universel.

Le sociologue peut toutefois se donner comme individu-type - comme homo sociologicus - un être de raison et de passion; il ne s'agit pas de renoncer aux avantages incontestables de la modélisation mais bien de remplacer un type par un autre $^{12}$. Il sera lors amené à admettre que ce qui ne se démontre pas est socialement tout aussi important - voire plus important - que ce qui peut faire l'objet d'une procédure de vérification. Si, en outre, le sociologue est conscient du «cercle piagétien » sujet-objet qui le lie intimement à la société dont il fait partie, il acceptera sans nul doute de s'appliquer les mêmes caractéristiques qu'à ses semblables dont il fait profession d'étudier le comportement: il ne peut que s'accepter lui aussi comme un être de raison et de passion, c'est-à-dire comme un individu dont les motivations et les justifications ne peuvent pas en toute occasion prétendre à l'approbation d'un auditoire universel. Son idéal d'objectivité scientifique ne le

A ce sujet, voir mes articles récents, consacrés à la dégradation de l'institution universitaire (Coenen-Huther, 2000 et 2001a).

12 Le type dominant, fondé sur le dualisme cartésien, a clairement montré ses limites dans la façon dont il a longtemps empêché la médecine de prendre en compte les interactions subtiles entre les aspects psychiques et organiques de la maladie (Voir à ce sujet: Damasio, 1994, 1995, Chap. 11, La passion fondant la raison). 
dispense pas du choix entre agir moralement ou pas. Et ce qu'il percevra comme l'action morale échappera à tout essai de réduction du normatif au cognitif. Dans un rôle d'enseignant, le sociologue ne peut qu'être confronté de nos jours à des jeunes gens tentés de remettre en question les règles qu'on leur impose, en prenant prétexte de la relativité de tout aménagement institutionnel. Il pourra rétorquer alors en toute tranquillité d'esprit, en ayant conscience d'avoir pour lui la logique et le bon sens, qu'il n'appartient pas aux bénéficiaires de droits-créances de définir eux-mêmes l'étendue de ces droits lorsque ceux-ci sont précisément la conséquence d'un état de vulnérabilité et d'immaturité. C'est cet état de vulnérabilité et d'immaturité se prolongeant de plus en plus tard de nos jours qui empêche de traiter les bénéficiaires des droits-créances comme des sujets de droit à part entière. Voilà un principe à portée tout à fait générale qui relève d'une sociologie formelle «style Simmel» et qui résiste à la relativisation socioculturelle. Quelle que soit l'époque, quelle que soit la culture, l'action éducative est basée sur le savoir et sur le pouvoir. Elle doit s'assumer comme telle et se soustraire à une contestation relativisante dont les effets pervers se manifestent déjà sous la forme d'une juridicisation excessive.

Mais soustraire les rapports entre générations au juridisme, ce n'est en aucune façon réduire le champ de nos responsabilités à l'égard des jeunes. Ceux-ci doivent pouvoir espérer bien davantage que les droits-libertés qu'on leur concède de manière tout à fait inconsidérée. Ils doivent pouvoir compter sur un soutien intellectuel et moral qui échappe largement à la perspective juridique mais qui est la concrétisation de ce qu'on peut appeler une éthique de sollicitude (Renaut, 2002, p. 367). La sollicitude «désigne ce sentiment de responsabilité» qu'on éprouve envers autrui, «y compris en l'absence de toute revendication de sa part au nom d'un quelconque droit » (Ibid.). C'est elle qui fonde la relation de maître à disciple, seul principe hiérarchique légitime en milieu universitaire. C'est précisément cette relation de maître à disciple qui est mise en péril à l'heure actuelle par un juridisme envahissant dont les conséquences s'avèrent proprement catastrophiques. Les manifestations empiriquement observables d'une telle éthique de sollicitude sont bien évidemment liées à une culture et à une époque: si ce n'était pas le cas, elles ne pourraient être ni comprises ni même perçues par ceux qui en sont l'objet. Mais son existence même est de tous les temps. Elle ne trouve pas son origine dans notre animalité mais bien dans la rupture avec la nature déjà mise en évidence dans un lointain passé par les Sophistes. La mettre en pratique, c'est tourner le dos à une parodie de démocratie qui décourage l'action éducative; c'est également en finir avec l'usage souvent abusif des prérogatives de la fonction professorale. C'est faire œuvre morale sans pour autant renoncer aux acquis majeurs de la sociologie: ceux qui portent sur les compatibilités et incompatibilités structurelles propres à tout système social.

Département de sociologie

Université de Genève 


\section{RÉFÉRENCES BIBLIOGRAPHIQUES}

Aron, Raymond (1981), Le spectateur engagé. Entretiens avec Jean-Louis Missika et Dominique Wolton, Paris, Julliard.

Aron, Raymond (1983), Mémoires, 50 ans de réflexion politique, Paris, Julliard.

Baverez, Nicolas (1993), Raymond Aron. Un moraliste au temps des idéologies, Paris, Flammarion.

Berthelot, Jean-Michel (1996), Les vertus de l'incertitude. Le travail de l'analyse dans les sciences sociales, Paris, Presses Universitaires de France.

Boudon, Raymond (1984), L'idéologie. L'origine des idées reçues, Paris, Fayard.

Boudon, Raymond (1995), Le juste et le vrai. Etudes sur l'objectivité des valeurs et de la connaissance, Paris, Fayard.

Boudon, Raymond (1999), Le sens des valeurs, Paris, Quadrige / Presses Universitaires de France.

Boudon, Raymond (2002), Déclin de la morale? Déclin des valeurs?, Paris, Presses Universitaires de France.

Bouthoul, Gaston (1949), Traité de sociologie, Paris, Payot, 2e édition.

Coenen-Huther, Jacques (1969), «La sociologie au service de l'administration publique», in: Res Publica, Revue de l'Institut Belge de Science Politique, XI, 2, pp. 309-326.

Coenen-Huther, Jacques (1979), «Professionnalisation de la sociologie et recherche appliquée», in: Revue Suisse de Sociologie, 5 (1), pp. 21-34.

Coenen-Huther, Jacques (1987), «Sociology and Decision-Making », in: Social Policy and Administration, Oxford, Vol. 21 (2), Summer, pp. 187-199.

Coenen-Huther, Jacques (1996), «L'observation participante et l'épistémologie piagétienne», in: Revue Européenne des Sciences Sociales, Tome XXXIV, No 106, pp. 133-140.

Coenen-Huther, Jacques (1997), Tocqueville, Paris, Presses Universitaires de France, Coll. «Que saisje?».

Coenen-Huther, Jacques (1998), «La sociologie face au changement social», in: Revue Européenne des Sciences Sociales, Tome XXXVI, No 110, pp. 121-134.

Coenen-Huther, Jacques (2000), «Les sociologues et la crise de l'Université: peur, aveuglement ou complicité?», in: Revue Européenne des Sciences Sociales, Tome XXXVIII, No 119, pp. 89-102.

Coenen-Huther, Jacques (2001a), «Les acteurs et les conséquences inattendues de leurs actions: le cas de l'université», in: Revue Européenne des Sciences Sociales, Tome XXXIX, No 121, pp. 111124.

Coenen-Huther, Jacques (2001b), «Sens moral ou raisons fortes?» in: L'Année sociologique, Vol. 51, No 1, pp. 233-254.

Coenen-Huther, Jacques (2002), «La sociologie des sciences entre positivisme et sociologisme», in: Revue Européenne des Sciences Sociales, Tome XL, No 124, pp. 219-231.

Damasio, Antonio R. (1994), Descartes'Error. Emotion, Reason and the Human Brain. New York, A. Grosset/Putnam Books. Tr. fr. L'erreur de Descartes. La raison des émotions, Paris, Odile Jacob, 1995.

Dupréel, Eugène (1932), Traité de morale, Bruxelles, Editions de la Revue de l'Université de Bruxelles.

Dupréel, Eugène (1939), Esquisse d'une philosophie des valeurs, Paris, Alcan.

Dupréel, Eugène (1949), Essais pluralistes, Paris, Presses Universitaires de France.

Ferry, Luc (2002), Qu'est-ce qu'une vie réussie? Essai, Paris, Grasset.

Ferry, Luc et Vincent, Jean-Didier (2000), Qu'est-ce que l'homme? Sur les fondamentaux de la biologie et de la philosophie, Paris, Odile Jacob.

Lassave, Pierre (2002), Sciences sociales et littérature. Concurrence, complémentarité, interférences. Paris, Presses Universitaires de France, Coll. «Sociologie d'aujourd'hui».

Lawick-Goodall, Jane Van (1973), «Cultural Elements in a Chimpanzee Community», in E. W. Menzel, Ed., Precultural Primate Behavior, Bâle, S. Karger, pp. 144-148. 
Lemel, Yannick (1991), Stratification et mobilité sociale, Paris, Armand Colin.

Lestel, Dominique (2001), Les origines animales de la culture, Paris, Flammarion.

Pascal, Blaise (†1670), Pensées, Genève, Skira, édition 1943.

Perelman, C. et Olbrechts-Tyteca, L. (1970), Traité de l'argumentation. La nouvelle rhétorique, Bruxelles, Editions de 1'Institut de Sociologie.

Renaut, Alain (2002), La libération des enfants. Contribution philosophique à une histoire de l'enfance, Paris, Bayard, Calmann-Lévy.

Revel, Jean-François (2002), L'obsession anti-américaine, Paris, Plon.

Riesman, David et al. (1950), The Lonely Crowd, New Haven, Yale University Press.

Simmel, Georg (1907), Philosophie des Geldes, Berlin, Duncker und Umblot. Tr. fr. Philosophie de l'argent, Paris, Quadrige / Presses Universitaires de France.

Tocqueville, Alexis de $(† 1893,1964)$, Souvenirs, Oeuvres complètes, tome XII, Paris, Gallimard. Unamuno, Miguel de (1917), Le sentiment tragique de la vie, traduit de l'espagnol (original 1912), Paris, Editions de la Nouvelle Revue Française.

Wilson, J. Q. (1993), The Moral Sense, New York, MacMillan/The Free Press. 